\title{
Inactivation of Clostridium sporogenes in Clover Honey by Pulsed UV-light Treatment
}

\author{
Sara L. Hillegas ${ }^{1}$ and Ali Demirci ${ }^{1,2^{*}}$ \\ ${ }^{1}$ Department of Agricultural and Biological Engineering, \\ ${ }^{2}$ The Huck Institutes for Life Sciences \\ The Pennsylvania State University, \\ University Park, Pennsylvania 16802 \\ *Author for correspondence: Tel: (814) 863-1098; Fax: (814) 863-1013; \\ e-mail: demirci@psu.edu
}

\begin{abstract}
Honey has naturally low levels of microorganisms due to its low water content and high concentration of sugars. However, it has been known to contain the spores of yeast, mold, and bacteria resulting in spoilage of the product. Clostridium botulinum, is associated with honey and is known to cause a disease called botulism. Heat pasteurization, pressure treatment, and irradiation have all been studied as methods of killing the spores, but have been found lacking. Pulsed Ultraviolet (UV)-Light Sterilization is a novel processing method used for microbial inactivation. For the experimentation, spores of $C$. sporogenes were prepared from cooked meat medium cultures grown in an anaerobic jar for 7 days at $37^{\circ} \mathrm{C}$. Honey was inoculated with $0.1 \mathrm{ml}$ of C. sporogenes spore suspension per $20 \mathrm{~g}$ honey, and mixed well. Pulsed light was applied to the inoculated honey using the SteriPulse XL ${ }^{\circledR}$ 3000 Pulsed Light Sterilization System provided by NASA Commercial Food Technology Center. The following parameters were analyzed: 1) the number of pulses 2) the distance between the food product and lamp 3) depth of honey. Treated and untreated (control) honey was analyzed for C. sporogenes. Increasing the number of pulses (or treatment time) also appears to be effective at inactivating the spores. When the depth of honey was decreased from 8 to $2 \mathrm{~mm}$ at the $20 \mathrm{~cm}$ shelf height, the spore kill increased for the same number of pulses. Even though varying these three parameters enhanced the inactivation of $C$. sporogenes up to $90 \%$, it failed to inactivate the spores completely due to insufficient penetration of UV-light into honey.
\end{abstract}

Keywords. Clover honey, Clostridium sporogenes, inactivation, decontamination, pulsed ultraviolet light 


\section{INTRODUCTION}

Honey is a food product that has naturally low levels of microorganisms due to its low water content and high concentration of sugars (Huhtanen, 1991). However, honey has been known to carry the spores of yeast, mold, and bacteria. This may occur in nature from dust, dead larvae or during the processing of honey. Although the microbes have difficulty growing in honey, they can survive and be transported to a new product that is mixed with the honey, resulting in growth and spoilage of the product (Snowdon, 1999). One bacterium in particular, Clostridium botulinum, is commonly found in honey and is known to cause botulism and infant botulism. In the United States, there is an average of 42 cases per year of infant botulism, and 33\% of these infants have consumed honey (Huhtanen, 1991). Therefore, parents are advised not to feed honey to children under the age of 2 years. C. botulinum is found naturally in soil and dust, so the general population is exposed to small concentrations of this spore on a daily basis. Over time, the human body will develop immunity to this bacterial toxin, but an infant body has not yet built up this immunity and the danger of contracting botulism from contaminated honey still exists. Clostridium sporogenes is in the same family as C. botulinum and has similar properties of heat resistance, light resistance, etc. (Welt et al., 1994).

Several methods of inactivating C. botulinum and C. sporogenes bacteria have been investigated. Welt et al. (1994) compared the inactivation of $C$. sporogenes with microwave radiation vs. conventional heating. No difference was found between the two methods, but thermal processing can alter the color, flavor, or nutritional content of the honey. Gamma radiation has been found to reduce the number of $C$. botulinum spores, but as the water content of the honey decreases, the resistance to radiation increases (Huhtanen, 1991). Hydrostatic pressure treatment has been used in conjunction with irradiation. It was found that high levels of hydrostatic pressure could reduce the irradiation dose needed to extend the shelf life of food (Crawford et al., 1996).

Ultraviolet light is an electromagnetic radiation in the spectral region, which is classified into three wavelength ranges: UV-C (100 nm - $280 \mathrm{~nm})$; UV-B (280 nm - $315 \mathrm{~nm})$; and UV-A (315 $\mathrm{nm}-400 \mathrm{~nm}$ ). UV-C light posses germicidal properties. It deactivates the DNA of bacteria, viruses and other pathogens and thus destroys their ability to multiply and cause disease. Specifically, UV-C light causes damage to the nucleic acid of microorganisms by formation of lethal thymine dimers on the microbial DNA (Bank et al., 1990; Jay, 1997). The formation of such bonds prevents the DNA from being unzipped for replication, and the organism is unable to reproduce. Ultraviolet technology is a non-chemical approach to disinfection. In this method of disinfection, nothing is added which makes this process simple, inexpensive and requires very low maintenance.

The conventional systems produce continuous UV-light with a power dissipation in the range of 100 to $1,000 \mathrm{~W}$. Generating these high intensity energy density levels with continuous UV-light can be costly to the user, which demands that systems be designed for the maximum conversion and collection efficiency of UV radiation. However, pulsed UV systems can dissipate many

S. Hillegas and A. Demirci. "Inactivation of Clostridium sporogenes in Clover Honey by Pulsed UV-light Treatment". Agricultural Engineering International: the CIGR Journal of Scientific Research and Development. Manuscript FP 03 009. Vol. V. December 2003. 
megawatts of electrical power in the light source. Therefore, a modest energy input (e.g., 3 Joules $/ \mathrm{cm}^{2}$ ) can yield high peak power dissipation ( 35 MW) (MacGregor et al., 1997; McDonald et al., 2000). The pulsed light flashes are created by compressing electrical energy into short pulses and using these pulses to energize an inert gas lamp. The lamp emits an intense flash of light with a duration of a few hundred microseconds. Because the lamp can be flashed many times per second, only few flashes are required to produce a high level of microbial kill.

Pulsed UV-light system can be considered a powerful tool for inactivation of microorganisms on food surfaces. Rowan et al. (1999) reported $\sim 6 \log _{10}$ reduction of Listeria monocytogenes, Escherichia coli O157:H7, Salmonella Enteritidis, Pseudomonas aeruginosa, Bacillus cereus, and Staphylococcus aureus which are seeded on agar plates by using pulsed UV light with 200 light pulses (pulse duration, $100 \mathrm{~ns}$ ). Also, McDonald et al. (2000) demonstrated inactivation of Bacillus subtilis spores by pulsed UV-light which resulted nearly three orders of magnitude of enhanced inactivation over continuous UV-light. All these limited studies clearly indicate the potential of pulsed UV-light systems with a modest energy input. In 1999, pulsed light treatment of food was approved by FDA (Federal Register, 1999). In this study, the pulsed UV-light treatment has been evaluated for decontamination of honey. The objective of this study is to determine the effect of number of pulses, distance from UV lamp, and depth of penetration during pulsed UV-light treatment of honey on inactivation of Clostridium sporogenes.

\section{MATERIALS AND METHODS}

\section{Microorganism.}

Clostridium sporogenes was obtained from the Food Microbiology Culture Collection in the Department of Food Science at the Pennsylvania State University. A C. sporogenes culture was grown statically in cooked meat medium (Difco Laboratories, Detroit, Mich.) in an anaerobic medium at $37^{\circ} \mathrm{C}$ for $24 \mathrm{~h}$ and stored in $10 \%$ glycerol at $-80^{\circ} \mathrm{C}$. Spores of $C$. sporogenes were prepared from cooked meat medium cultures grown in an anaerobic jar for 7 days at $37^{\circ} \mathrm{C}$. Vegetative cells were destroyed by heating the culture in an $80^{\circ} \mathrm{C}$ water bath for $20 \mathrm{~min}$. Then, the culture was centrifuged at 3,300 $\mathrm{x} g$ for $20 \mathrm{~min}$, and diluted with $0.1 \%$ sterile peptone water to obtain a concentration of approximately $10^{8}$ spores per $\mathrm{ml}$. The spore count was determined by pour plating the spore solution on Anaerobic agar (Difco) after serial dilution in $0.1 \%$ sterile peptone water. The prepared spore suspension was stored at $4^{\circ} \mathrm{C}$ until use.

\section{Honey inoculation.}

Clover honey was purchased from the Penn State University Creamery. Honey was weighed in a sterile beaker covered with aluminum foil, and inoculated with spore suspension $(0.1 \mathrm{ml}$ per $20 \mathrm{~g}$ of honey) to achieve about $6 \log _{10} \mathrm{CFU} / \mathrm{ml}$. The inoculated honey was stirred for 5 minutes to ensure homogenous distribution of spores. Then, one gram of honey was transferred into a sterile test tube containing $10 \mathrm{ml}$ of sterile $0.1 \%$ peptone water. This sample of contaminated honey served as the untreated sample for calculating the spore reduction after treatment. All sampling

S. Hillegas and A. Demirci. "Inactivation of Clostridium sporogenes in Clover Honey by Pulsed UV-light Treatment". Agricultural Engineering International: the CIGR Journal of Scientific Research and Development. Manuscript FP 03 009. Vol. V. December 2003. 
was done in triplicates. After serial dilutions, the spore suspensions were pour plated with Anaerobic Agar.

\section{Treatment with pulsed UV-light.}

Pulsed UV-light was generated by the SteriPulse ${ }^{\circledR}-X L 3000$ Pulsed Light Sterilization System (Xenon Corp., MA) with an equipment setting of 3 pulses/sec. Inoculated honey samples were placed in the pulse UV chamber where they were exposed to pulses of UV-light. The energy released from the UV strobe was $5.6 \mathrm{~J} / \mathrm{cm}^{2}$ per pulse when the input voltage was $3,800 \mathrm{~V}$.

The effect of honey thickness (i.e., depth of honey) was determined on the treatment effectiveness with 20 and $5 \mathrm{~g}$ samples of inoculated honey in aluminum weigh boat with $50 \mathrm{~mm}$ diameter at middle distance from the UV lamp $(20 \mathrm{~cm})$. These weights corresponded with thicknesses of $8 \mathrm{~mm}$ and $2 \mathrm{~mm}$, respectively. At each thickness the effect of number of pulses was investigated for treatment times up to $180 \mathrm{~s}$, which were controlled using a timer built into the control panel of the pulsed UV-light system.

In order to study the effect of distance from the UV strobe and treatment time on inactivation of C. sporogenes, $5 \mathrm{~g}$ of inoculated honey in the aluminum weigh boat were placed on the tray in the treatment chamber at three different distances from the UV lamp: 8, 13, $20 \mathrm{~cm}$. At each distance, the effect of number of pulses was investigated up to $135 \mathrm{~s}$. For the pulsed UV-light treatments, the temperature profiles of uninoculated honey at various depths and distances were monitored using a type K thermocouple (Omegaette HH306, Omega Engineering Inc., Stamford, $\mathrm{CT})$. Triplicate temperature measurements were taken using the thermocouple placed $1 \mathrm{~mm}$ below the surface of honey.

\section{Analysis.}

Pulsed UV treated and untreated honey samples were analyzed for C. sporogenes.

Approximately $1 \mathrm{~g}$ of the treated honey sample was weighed into a sterile test tube and dissolved $10 \mathrm{ml}$ of $0.1 \%$ peptone water. After serial dilutions, each dilution was pour plated with Anaerobic Agar (Difco, Detroit, MI). Then the plates were incubated at $37^{\circ} \mathrm{C}$ for 48 hours in an anaerobic jar, and colonies were enumerated. All sampling was done in triplicates

\section{RESULTS AND DISCUSSION}

Honey inoculated with Clostridium sporogenes spores was placed in aluminum dishes and treated with pulsed UV-light treatment. The number of pulses, the distance between honey and lamp, and depth of honey were investigated. The results from each experiment have been summarized in Table 1. For each set of parameters, the percent reduction was calculated.

S. Hillegas and A. Demirci. "Inactivation of Clostridium sporogenes in Clover Honey by Pulsed UV-light Treatment". Agricultural Engineering International: the CIGR Journal of Scientific Research and Development. Manuscript FP 03 009. Vol. V. December 2003. 
Although the results varied slightly between repetitions of the same combination of parameters, several trends can be noted from the data obtained. By examining the data at a shelf height of 20 $\mathrm{cm}$ and $8 \mathrm{~mm}(20 \mathrm{~g})$ depth of honey, it can be noted that increasing the number of pulses (which also means increasing the treatment time) will increase the percent reduction. The results show an increase from $0 \%$ reduction at 15 and 135 pulses ( 5 and 45 seconds) to $89.4 \%$ reduction at 540 pulses (3 minutes).

Table 1. Inactivation of C. sporogenes in honey.

\begin{tabular}{|c|c|c|c|c|c|}
\hline $\begin{array}{c}\text { Shelf Height } \\
(\mathbf{c m})\end{array}$ & $\begin{array}{c}\text { Treatment } \\
\text { Time } \\
(\mathbf{s e c})\end{array}$ & $\begin{array}{c}\text { Pulse } \\
\text { (Number) }\end{array}$ & $\begin{array}{c}\text { Depth of } \\
\text { Honey } \\
(\mathbf{m m})\end{array}$ & $\begin{array}{c}\text { Initial Count } \\
\text { (log/g) }\end{array}$ & $\begin{array}{c}\text { Reduction } \\
(\mathbf{\%})\end{array}$ \\
\hline 20 & 5 & 15 & 8 & 6.25 & 0.0 \\
\hline 20 & 45 & 135 & 8 & 6.25 & 0.0 \\
\hline 20 & 90 & 270 & 8 & 6.32 & 22.1 \\
\hline 20 & 135 & 405 & 8 & 6.32 & 14.2 \\
\hline 20 & 180 & 540 & 8 & 6.32 & 89.4 \\
\hline 20 & 45 & 135 & 2 & 6.15 & 39.5 \\
\hline 20 & 90 & 270 & 2 & 6.15 & 28.9 \\
\hline 20 & 135 & 405 & 2 & 6.15 & 73.9 \\
\hline 13 & 45 & 135 & 2 & 6.24 & 49.4 \\
\hline 13 & 60 & 180 & 2 & 6.24 & 80.8 \\
\hline 8 & 45 & 135 & 2 & 6.24 & 87.6 \\
\hline
\end{tabular}

The tendency for increased reduction when the depth of honey was decreased from $8 \mathrm{~mm}$ to 2 $\mathrm{mm}$ can be seen by comparing the data at a constant shelf height of $20 \mathrm{~cm}$ and number of pulses ranging from 135, 270, and 405. When the depth of honey was changed from $8 \mathrm{~mm}$ to $2 \mathrm{~mm}$, an increase in reduction becomes evident. At the $8 \mathrm{~mm}$ depth, 135, 270, and 405 pulses demonstrated $0,22.1$, and $14.2 \%$ reduction, respectively. In comparison, at the $2 \mathrm{~mm}$ depth, 135, 270 , and 405 pulses demonstrated $39.5,28.9$, and $73.9 \%$ reductions, respectively.

The trend that emerges from decreasing the shelf height, or decreasing the distance between the honey and the UV light, can be seen by examining the percent reduction for different shelf heights at the same number of pulses and the same depth of honey. At a shelf height of $20 \mathrm{~mm}$,

S. Hillegas and A. Demirci. "Inactivation of Clostridium sporogenes in Clover Honey by Pulsed UV-light Treatment". Agricultural Engineering International: the CIGR Journal of Scientific Research and Development. Manuscript FP 03 009. Vol. V. December 2003. 
135 pulses, and a $2 \mathrm{~mm}$ depth of honey, the spore reduction was $39.5 \%$. When the shelf was at $13 \mathrm{~cm}, 135$ pulses, and a $2 \mathrm{~mm}$ depth of honey, the spore reduction was $49.4 \%$. Finally, when the shelf was at the $8 \mathrm{~cm}$ height, 135 pulses, and $2 \mathrm{~mm}$ depth of honey, the spore reduction was $87.6 \%$. This increasing amount of reduction demonstrates that decreasing the shelf height (decreasing the distance between the honey and the UV lamp) increases the percent spore reduction. Upon the completion of the experimental procedure, it was of interest to determine the temperatures that were achieved at the different shelf heights and treatment times. In general, raising the shelf height resulted in higher temperatures especially for $2 \mathrm{~mm}$ thickness (Fig. 1).

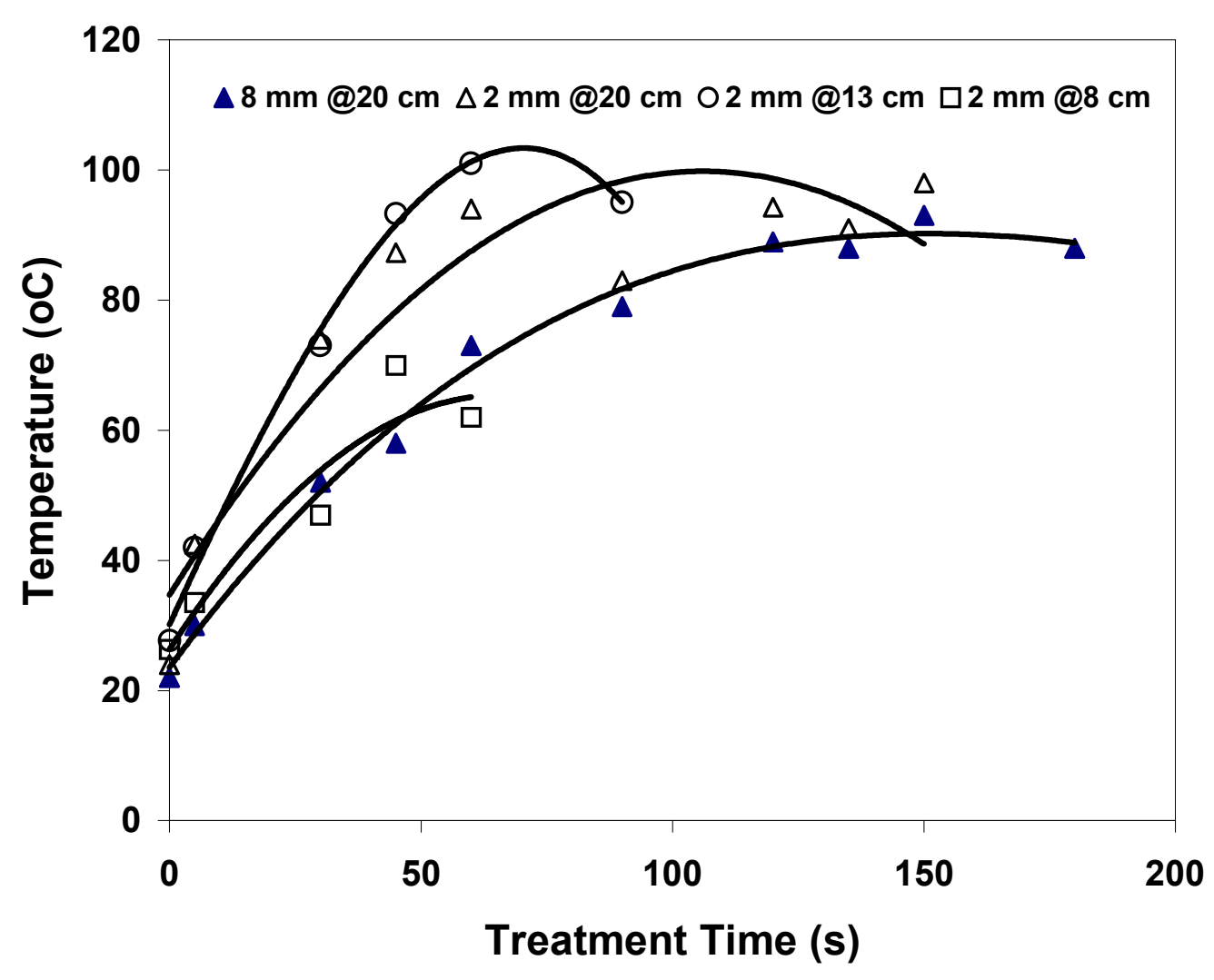

Figure 1. Temperature profiles for the various honey thicknesses and distances from UV lamp.

\section{CONCLUSION}

Raising the shelf (or decreasing the distance between the honey surface and the UV-lamp) demonstrated an increase in spore reduction. Increasing the number of pulses (or treatment time) also appears to be effective at inactivating the spores. When the depth of honey was decreased

S. Hillegas and A. Demirci. "Inactivation of Clostridium sporogenes in Clover Honey by Pulsed UV-light Treatment". Agricultural Engineering International: the CIGR Journal of Scientific Research and Development. Manuscript FP 03 009. Vol. V. December 2003. 
from 8 to $2 \mathrm{~mm}$ at the $20 \mathrm{~cm}$ shelf height, the spore kill increased for the same number of pulses. Even though varying these three parameters enhanced the percent inactivation of $C$. sporogenes, it failed to inactivate the spores completely. It appears that the UV-light has a limited penetration in the honey. The heat generated within the Pulsed UV-light does not appear to have a synergistic effect on the inactivation of $C$. sporogenes in honey.

\section{ACKNOWLEDGMENTS}

Pulsed UV-light sterilization system was provided by NASA Food Technology Commercial Space Center equipment grant. This research was funded in part by the Undergraduate Research Program of the College of Agricultural Sciences at the Pennsylvania State University and the Pennsylvania Agricultural Experiment Station.

\section{REFERENCES}

Bank, H.L., John, J., Schmehl, M.K., and Dratch, R.J. 1990. Bacteriocidal effectiveness of modulated UV light. Appl. Environ. Microbiol. 56:3888-3889.

Crawford, Y.J., Murano, E.A., Olson, D.G., Shenoy, K. 1996. Use of high hydrostatic pressure and irradiation to eliminate Clostridium sporogenes spores in chicken breast. J. Food Prot. 59:711-715.

Huhtanen, C.N. 1991. Gamma radiation resistance of Clostridium botulinum 62A and Bacillus subtilis spores in honey. J. Food Prot. 54:894-896.

Jay, J.M. 1997. Radiation preservation of foods and nature of microbial radiation resistance. In Modern food microbiology. pp. 304-323. Chapman and Hall, New York, N.Y.

MacGregor, S.J., Turnbull, S.M., Tuema, F.A., and Farish, O. 1997. Factors affecting and methods of improving the pulse repetition frequency of pulse-charged and de-charged high pressure gas switches. IEEE Trans Plasma Sci. 25:110-117.

McDonald K. F, Curry, R.D, Clevenger, T.E., Unklesbay, K., Eisenstark, A., Golden, J., Morgen, R.D. 2000. A comparison of pulsed and continuos ultraviolet light sources for the decontamination of surfaces. IEEE Trans Plasma. Sci. 28:1581-1587.

Rowan, N.J., MacGregor, S.J., Anderson, J.G., Fouracre, R.A., McIlvaney, L., and Farsih, O. 1999. Pulsed-light inactivation of food-related microorganisms. Appl. Environ. Microbiol. 65:1312-1315.

Snowdon, J.A. 1999. The microbiology of honey. Am. Bee J. 139:51-60.

Welt, B.A., Tong, C.H., Rossen, J.L., and Lund, D.B. 1994. Effect of microwave radiation on inactivation of Clostridium sporogenes. Appl. Environ. Microbiol. 60:482-488.

S. Hillegas and A. Demirci. "Inactivation of Clostridium sporogenes in Clover Honey by Pulsed UV-light Treatment". Agricultural Engineering International: the CIGR Journal of Scientific Research and Development. Manuscript FP 03 009. Vol. V. December 2003. 